Supplement of Geosci. Model Dev. Discuss., 8, 10411-10454, 2015

http://www.geosci-model-dev-discuss.net/8/10411/2015/

doi:10.5194/gmdd-8-10411-2015-supplement

(C) Author(s) 2015. CC Attribution 3.0 License.

(c) (i)

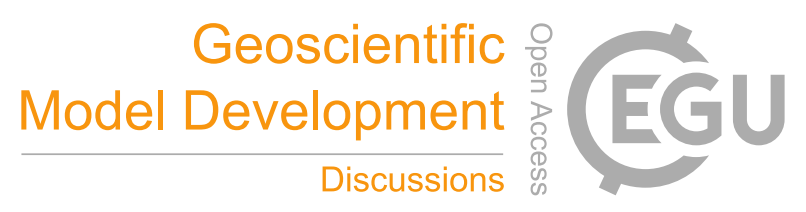

Supplement of

\title{
An integrated Dissolved Organic Carbon Dynamics Model (DOCDM 1.0): model development and a case study in the Alaskan Yukon River Basin
}

\author{
X. Lu and Q. Zhuang \\ Correspondence to: Q. Zhuang (qzhuang@purdue.edu)
}

The copyright of individual parts of the supplement might differ from the CC-BY 3.0 licence. 


\section{Supplement A}

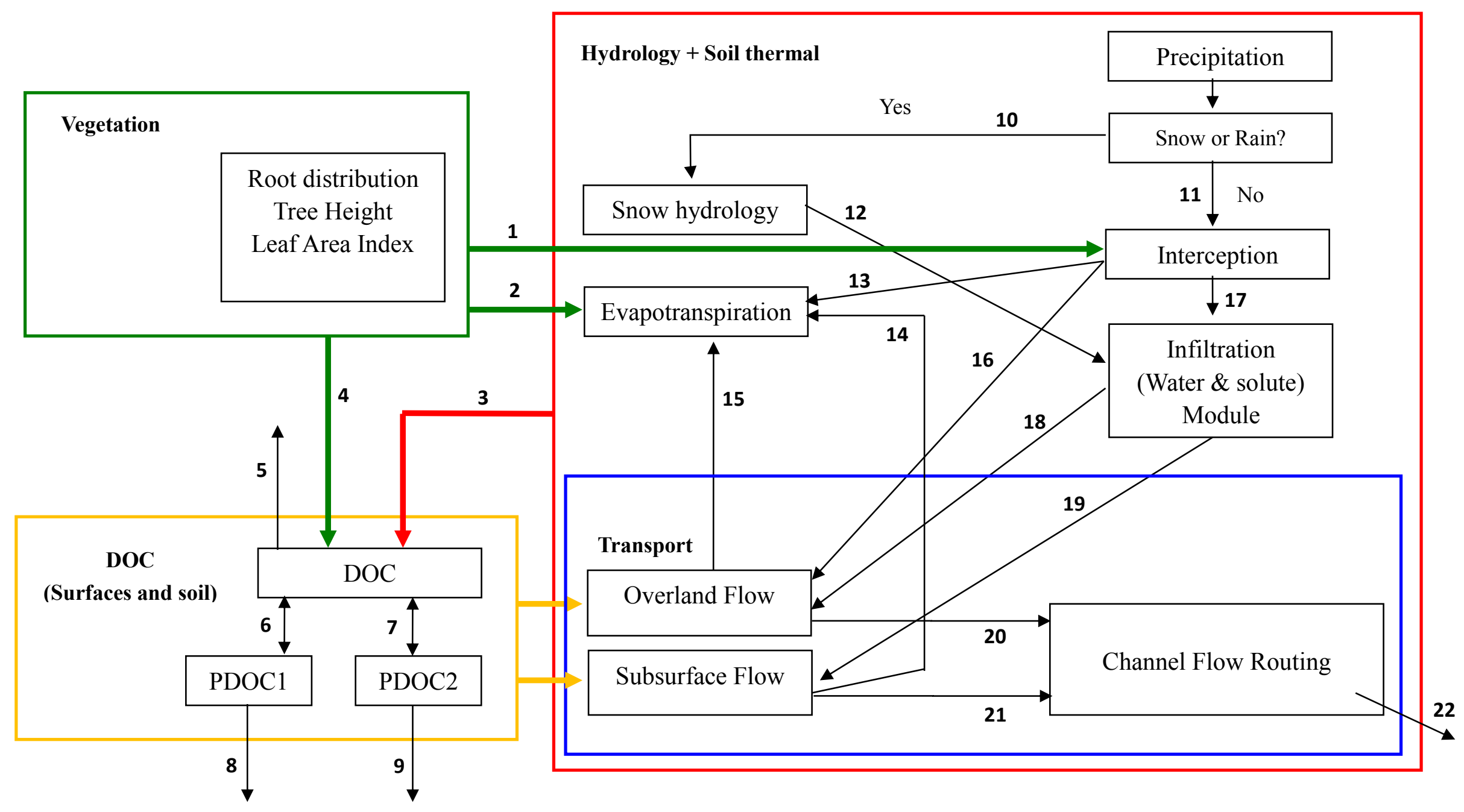


1: Vegetation variables, such as LAI, used in the snowfall and rainfall interception algorithm.

2: Vegetation variables, such as LAI, stomatal_conductance and root depth, used in the snowfall and rainfall interception algorithm.

3: Soil temperature, moisture information required in the DOC production and mineralization.

4: Soil organic layer distribution information used in the DOC production.

5: The mineralization of DOC.

6: Conversion (absorption and desorption) between DOC and PDOC1.

7: Conversion (absorption and desorption) between DOC and PDOC2.

8: The mineralization of PDOC1.

9: The mineralization of PDOC2.

10: If snow, the method to estimate snowpack dynamic is applied.

11: To calculate how much snow and rain is intercepted by vegetation canopy.

12: Snowmelt water used in the infiltration method.

13: The amount of intercepted precipitation used in the evapotranspiration algorithm.

14: Soil moisture loss in the evapotranspiration.

15: Evapotranspiration on the overland flow.

16: ThroughFall precipitation joins overland flow.

17: ThroughFall precipitation may infiltrate into soil.

18: Horton overland flow or saturation overland flow.

19: Groundwater comes from infiltrated water.

20: Overland flow enters channel networks

21: Subsurface flow enter channel networks

22: Channel flow levels at the outlets. 


\section{Supplement B}

Two types of upper boundary conditions are defined: specified flux when there is no surface ponding, and specified pressure head when there is surface ponding. For the first condition, the specified flux is the sum of rainfall rate and the soil evaporation rate. In the case of a head boundary condition, the pressure in the top grid is equal to the depth of the ponding water. The top boundary conditions may be changed from specified flux into specified pressure head, and vice versa, during the iterative solution of the Richards equation (van Dam and Feddes, 2000). Specifically, when a flux boundary condition is initially specified, a source term (equal to the specified flux multiplied by the current time step) is added to the first non-boundary cell. New heads are calculated with this assumed source term. These heads are used to compute the inter-cell fluxes. If the calculated flux on the upper boundary of cell 1 is smaller than the specified flux, water ponds on the ground surface, and the upper boundary is changed to a specified head, the head being the depth of ponded water. Water that enters the top cell is infiltration. Any water that does not infiltrate becomes surface runoff which may join overland flow and be lost due to evaporation.

The lower boundary is at the bedrock, and $q_{b o t}=\rho * K_{b o t}$. If $\rho=1$, it means free drainage boundary condition. When there is no water table in the soil column, the lower boundary condition is a zero-head gradient. In practice, $\rho$ needs to be adjusted by comparison between simulated and measured stream flow. 

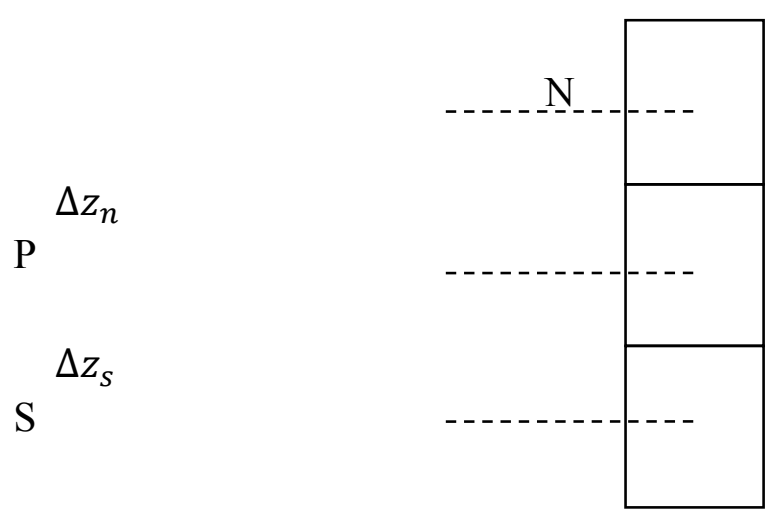

Fig. B.1 Geometric variables for a typical grid

Nodes in the domain may be identified by their $i$ grid indices. Figure B.1 is a detailed sketch of one grid as node $P$ in the domain. The $i-1$ and $i+1$ neighbors of $P$ are designated as $\mathrm{N}$ (upper) and $\mathrm{S}$ (lower) for north and south, respectively. The figure also defines the distance $\Delta z_{n}$ and $\Delta z_{s}$ between $P$ and its north and south neighbors. 
Equation $2 \mathrm{~b}$ in the main text can be discretized as:

$\left(\frac{d \theta}{d h}\right)_{i}^{n+1, m} * \frac{h_{i}^{n+1, m+1}-h_{i}^{n+1, m}}{\Delta t}-\frac{1}{(\Delta z)^{2}} *\left[K_{i+\frac{1}{2}}^{n+1, m} * h_{i+1}^{n+1, m+1}-K_{i+\frac{1}{2}}^{n+1, m} * h_{i}^{n+1, m+1}-K_{i-\frac{1}{2}}^{n+1, m} * h_{i}^{n+1, m+1}+K_{i-\frac{1}{2}}^{n+1, m} * h_{i-1}^{n+1, m+1}\right]=\frac{K_{i+\frac{1}{2}}^{n+1, m}-K_{i-\frac{1}{2}}^{n+1, m}}{\Delta z}-$ $\frac{\theta_{i}^{n+1, m}-\theta_{i}^{n}}{\Delta t}+S_{i}^{n+1}($ B. 1$)$

and can be further rearranged into

$$
\begin{gathered}
C_{i}^{n+1, m} * \frac{h_{i}^{n+1, m+1}-h_{i}^{n+1, m}}{\Delta t}-\frac{1}{(\Delta z)^{2}} *\left[K_{i+\frac{1}{2}}^{n+1, m} * h_{i+1}^{n+1, m+1}-K_{i+\frac{1}{2}}^{n+1, m} * h_{i}^{n+1, m+1}-K_{i-\frac{1}{2}}^{n+1, m} * h_{i}^{n+1, m+1}+K_{i-\frac{1}{2}}^{n+1, m} * h_{i-1}^{n+1, m+1}\right] \\
\left.=\frac{K_{i+\frac{1}{2}}^{n+1, m}-K_{i-\frac{1}{2}}^{n+1, m}}{\Delta z}-\frac{\theta_{i}^{n+1, m}-\theta_{i}^{n}}{\Delta t}+\mathrm{S}_{i}^{\mathrm{n}+1} \text { (B. } 1\right)
\end{gathered}
$$

Where $i, \Delta z$ and $h^{n}$ denote the spatial location, node size and the approximate value of $h$ at the $n t h$ discrete time interval, respectively, $\Delta t \equiv$ $t^{n+1}-t^{n}$ is the time step, $\left(\frac{d \theta}{d h}\right)_{i}^{n+1, m}$ and $K^{n+1}$ are specific moisture capacity and hydraulic conductivity values evaluated using $h^{n+1}$, respectively, and the solution is known at time $n$ and unknown at $n+1$. Since $\left(\frac{d \theta}{d h}\right)$ and $K$ are nonlinear functions of $h$, the Picard iteration $($ Celia et al., 1990) is introduced for each time step, and $m$ stands for iteration times in Eq. B.1. 

n: Time
$m$ : Iteration level
$i$ : Location
$N$ : Number of nodes
h: Head
$\Delta t$ : Time step
$\Delta z$ : The size of node
$C_{i}^{n+1, m}=\left(\frac{d \theta}{d h}\right)_{i}^{n+1, m}:$ Specific moisture capacity

$K$ : Hydraulic conductivity

From Eq. B.1, we have:

$$
\begin{gathered}
C_{i}^{n+1, m} * h_{i}^{n+1, m+1}-\frac{\Delta t}{(\Delta z)^{2}} *\left[K_{i+\frac{1}{2}}^{n+1, m} * h_{i+1}^{n+1, m+1}-\left(K_{i+\frac{1}{2}}^{n+1, m}+K_{i-\frac{1}{2}}^{n+1, m}\right) * h_{i}^{n+1, m+1}+K_{i-\frac{1}{2}}^{n+1, m} * h_{i-1}^{n+1, m+1}\right] \\
=\frac{\Delta t *\left(K_{i+\frac{1}{2}}^{n+1, m}-K_{i-\frac{1}{2}}^{n+1, m}\right)}{\Delta z}-\left(\theta_{i}^{n+1, m}-\theta_{i}^{n}\right)+C_{i}^{n+1, m} * h_{i}^{n+1, m}+\Delta t * S_{i}^{\mathrm{n}+1}
\end{gathered}
$$

It can be expressed with a matrix form:

$$
\left(\begin{array}{ccccc}
\beta_{1} & \gamma_{1} & 0 & 0 & 0 \\
\alpha_{2} & \beta_{2} & \gamma_{2} & 0 & 0 \\
\cdots & \cdots & \cdots & \cdots & \cdots \\
0 & 0 & 0 & \alpha_{N} & \beta_{N}
\end{array}\right) *\left[\begin{array}{c}
h_{1}^{n+1, m+1} \\
h_{2}^{n+1, m+1} \\
\vdots \\
h_{N}^{n+1, m+1}
\end{array}\right]=\begin{gathered}
f_{1} \\
f_{2} \\
\vdots \\
f_{N}
\end{gathered}
$$


We have:

$$
\begin{aligned}
& \alpha_{i}=-\frac{\Delta t}{(\Delta z)^{2}} * K_{i-\frac{1}{2}}^{n+1, m} \\
& \beta_{i}=C_{i}^{n+1, m}+\frac{\Delta t}{(\Delta z)^{2}} * K_{i+\frac{1}{2}}^{n+1, m}+\frac{\Delta t}{(\Delta z)^{2}} * K_{i-\frac{1}{2}}^{n+1, m} \\
& \gamma_{i}=-\frac{\Delta t}{(\Delta z)^{2}} * K_{i+\frac{1}{2}}^{n+1, m} \\
& f_{i}=\frac{\Delta t *\left(K_{i+\frac{1}{2}}^{n+1, m}-K_{i-\frac{1}{2}}^{n+1, m}\right)}{\Delta z}-\left(\theta_{i}^{n+1, m}-\theta_{i}^{n}\right)+C_{i}^{n+1, m} * \mathrm{~h}_{i}^{n+1, m}+\Delta t * \mathrm{~S}_{i}^{\mathrm{n}+1}
\end{aligned}
$$

\section{Boundary condition}

Equation B.1 can be also written as:

$$
\begin{aligned}
& C_{i}^{n+1, m} *\left(h_{i}^{n+1, m+1}-h_{i}^{n+1, m}\right)-\frac{\Delta t}{\Delta z} *\left[K_{i-\frac{1}{2}}^{n+1, m} *\left[\frac{h_{i-1}^{n+1, m+1}-H_{i}^{n+1, m+1}}{\Delta z}\right]-K_{i+\frac{1}{2}}^{n+1, m} *\left[\frac{H_{i}^{n+1, m+1}-H_{i+1}^{n+1, m+1}}{\Delta z}\right]\right]=\Delta t *\left[\frac{K_{i+\frac{1}{2}}^{n+1, m}-K_{i-\frac{1}{2}}^{n+1, m}}{\Delta z}\right]-\left(\theta_{i}^{n+1, m}-\right. \\
& \left.\theta_{i}^{n}\right)+\Delta t * S_{i}^{\mathrm{n}+1}(\mathrm{~B} .2) \\
& C_{i}^{n+1, m} *\left(h_{i}^{n+1, m+1}-h_{i}^{n+1, m}\right)+\left(\theta_{i}^{n+1, m}-\theta_{i}^{n}\right)+\Delta t * S_{i}^{\mathrm{n}+1}=\frac{\Delta t}{\Delta z} *\left[K_{i-\frac{1}{2}}^{n+1, m} *\left[\frac{h_{i-1}^{n+1, m+1}-h_{i}^{n+1, m+1}}{\Delta z}\right]+K_{i+\frac{1}{2}}^{n+1, m}-K_{i+\frac{1}{2}}^{n+1, m} *\left[\frac{h_{i}^{n+1, m+1}-h_{i+1}^{n+1, m+1}}{\Delta z}\right]-\right.
\end{aligned}
$$


$\left.K_{i-\frac{1}{2}}^{n+1, m}\right](\mathrm{B} .3)$

\section{Top boundary condition (i=1): specified flux $\boldsymbol{q}_{\text {surf }}$}

From Eq. B.3, we have:

$C_{i}^{n+1, m} *\left(h_{i}^{n+1, m+1}-h_{i}^{n+1, m}\right)+\left(\theta_{i}^{n+1, m}-\theta_{i}^{n}\right)+\Delta t * S_{i}^{n+1}=\frac{\Delta t}{\Delta z} *\left[q_{s u r f}-K_{i+\frac{1}{2}}^{n+1, m} *\left[\frac{h_{i}^{n+1, m+1}-h_{i+1}^{n+1, m+1}}{\Delta z}\right]-K_{i+\frac{1}{2}}^{n+1, m}\right]$

$C_{i}^{n+1, m} * h_{i}^{n+1, m+1}+\frac{\Delta t}{(\Delta z)^{2}} * K_{i+\frac{1}{2}}^{n+1, m} * h_{i}^{n+1, m+1}-\frac{\Delta t}{(\Delta z)^{2}} * K_{i+\frac{1}{2}}^{n+1, m} h_{i+1}^{n+1, m+1}$

$=C_{i}^{n+1, m} * h_{i}^{n+1, m}-\Delta t *\left[\frac{K_{i+\frac{1}{2}}^{n+1, m}}{\Delta z}\right]-\left(\theta_{i}^{n+1, m}-\theta_{i}^{n}\right)+\Delta t * S_{i}^{n+1}+\frac{\Delta t}{\Delta z} * q_{\text {surf }}$

$\alpha_{1}=0$

$\beta_{1}=C_{1}^{n+1, m}+\frac{\Delta t}{(\Delta z)^{2}} * K_{i+\frac{1}{2}}^{n+1, m}$

$\gamma_{1}=-\frac{\Delta t}{(\Delta z)^{2}} * K_{i+\frac{1}{2}}^{n+1, m}$ 
$f_{1}=\frac{\Delta t *\left(q_{\text {surf }}-K_{1+\frac{1}{2}}^{n+1, m}\right)}{\Delta z}-\left(\theta_{1}^{n+1, m}-\theta_{1}^{n}\right)+\Delta t * S_{i}^{n+1}+C_{1}^{n+1, m} * h_{1}^{n+1, m}$

\section{Top boundary condition (i=1): specified head $\boldsymbol{h}_{\text {surf }}$}

From Eq. B. 2 and by assuming $h_{i-1}^{n+1, m+1}=h_{\text {surf }}$, we have:

$$
\begin{gathered}
C_{i}^{n+1, m} *\left(h_{i}^{n+1, m+1}-h_{i}^{n+1, m}\right)-\frac{\Delta t}{(\Delta z)^{2}} *\left[K_{i-\frac{1}{2}}^{n+1, m} * h_{\text {surf }}-K_{i-\frac{1}{2}}^{n+1, m} * h_{i}^{n+1, m+1}-K_{i+\frac{1}{2}}^{n+1, m} * h_{i}^{n+1, m+1}+K_{i+\frac{1}{2}}^{n+1, m} * h_{i+1}^{n+1, m+1}\right] \\
=\Delta t *\left[\frac{\left(K_{i-\frac{1}{2}}^{n+1, m} / 2\right)-K_{i+\frac{1}{2}}^{n+1, m}}{\Delta z}\right]-\left(\theta_{i}^{n+1, m}-\theta_{i}^{n}\right)+\Delta t * S_{i}^{n+1} \\
\left(C_{i}^{n+1, m}+\frac{\Delta t}{(\Delta z)^{2}} * K_{i-\frac{1}{2}}^{n+1, m}+\frac{\Delta t}{(\Delta z)^{2}} * K_{i+\frac{1}{2}}^{n+1, m}\right) * h_{i}^{n+1, m+1}-\frac{\Delta t}{(\Delta z)^{2}} * K_{i+\frac{1}{2}}^{n+1, m} * h_{i+1}^{n+1, m+1} \\
=C_{i}^{n+1, m} * h_{i}^{n+1, m}+\frac{\Delta t}{(\Delta z)^{2}} * K_{i-\frac{1}{2}}^{n+1, m} * h_{\text {surf }}+\Delta t *\left[\frac{\left(K_{i-\frac{1}{2}}^{n+1, m} / 2\right)-K_{i+\frac{1}{2}}^{n+1, m}}{\Delta z}\right]-\left(\theta_{i}^{n+1, m}-\theta_{i}^{n}\right)+\Delta t * S_{i}^{n+1}
\end{gathered}
$$


$\alpha_{1}=0$

$\beta_{1}=C_{1}^{n+1, m}+\frac{\Delta t}{(\Delta z)^{2}} * K_{1-\frac{1}{2}}^{n+1, m}+\frac{\Delta t}{(\Delta z)^{2}} * K_{1+\frac{1}{2}}^{n+1, m}$

$\gamma_{1}=-\frac{\Delta t}{(\Delta z)^{2}} * K_{1+\frac{1}{2}}^{n+1, m}$

$f_{1}=C_{1}^{n+1, m} * h_{1}^{n+1, m}+\frac{\Delta t}{(\Delta z)^{2}} * K_{1-\frac{1}{2}}^{n+1, m} * h_{\text {surf }}+\Delta t *\left[\frac{0.5 * K_{1-\frac{1}{2}}^{n+1, m}-K_{1+\frac{1}{2}}^{n+1, m}}{\Delta z}\right]-\left(\theta_{1}^{n+1, m}-\theta_{1}^{n}\right)+\Delta t * S_{i}^{n+1}$

\section{Bottom boundary condition (i=N): specified flux $\boldsymbol{q}_{b o t}$}

From Eq. B.3, we have:

$C_{N}^{n+1, m} *\left(h_{N}^{n+1, m+1}-h_{N}^{n+1, m}\right)-\frac{\Delta t}{\Delta z} *\left[K_{N-\frac{1}{2}}^{n+1, m} *\left[\frac{h_{N-1}^{n+1, m+1}-h_{N}^{n+1, m+1}}{\Delta z}\right]+K_{N-\frac{1}{2}}^{n+1, m}-q_{b o t}\right]=-\left(\theta_{N}^{n+1, m}-\theta_{N}^{n}\right)+\Delta t * \mathrm{~S}_{i}^{\mathrm{n}+1}$

By rearranging:

$$
\begin{aligned}
C_{N}^{n+1, m} * h_{N}^{n+1, m+1}-\frac{\Delta t}{(\Delta z)^{2}} * K_{N-\frac{1}{2}}^{n+1, m} * h_{N-1}^{n+1, m+1}+\frac{\Delta t}{(\Delta z)^{2}} * K_{N-\frac{1}{2}}^{n+1, m} * h_{N}^{n+1, m+1} \\
=C_{N}^{n+1, m} * h_{N}^{n+1, m}+\Delta t *\left[\frac{K_{N-\frac{1}{2}}^{n+1, m}}{\Delta z}\right]-\left(\theta_{N}^{n+1, m}-\theta_{N}^{n}\right)+\Delta t * S_{i}^{n+1}-\frac{\Delta t}{\Delta z} * q_{b o t}
\end{aligned}
$$




$$
\begin{aligned}
& \alpha_{N}=-\frac{\Delta t}{(\Delta z)^{2}} * K_{N-\frac{1}{2}}^{n+1, m} \\
& \beta_{N}=C_{N}^{n+1, m}+\frac{\Delta t}{(\Delta z)^{2}} * K_{N-\frac{1}{2}}^{n+1, m} \\
& \gamma_{N}=0 \\
& f_{N}=C_{N}^{n+1, m} * h_{N}^{n+1, m}+\Delta t *\left[\frac{K_{N-\frac{1}{2}}^{n+1, m}}{\Delta z}\right]-\left(\theta_{N}^{n+1, m}-\theta_{N}^{n}\right)+\Delta t * S_{i}^{n+1}-\frac{\Delta t}{\Delta z} * q_{b o t}
\end{aligned}
$$

\section{Bottom boundary condition ( $\mathrm{i}=\mathrm{N})$ : specified head $\boldsymbol{h}_{b o t}$}

From Eq. B.2, we have: 


$$
\begin{gathered}
C_{N}^{n+1, m} *\left(h_{N}^{n+1, m+1}-h_{N}^{n+1, m}\right)-\frac{\Delta t}{(\Delta z)^{2}} *\left[K_{N-\frac{1}{2}}^{n+1, m} *\left(h_{N-1}^{n+1, m+1}-h_{N}^{n+1, m+1}\right)-K_{N+\frac{1}{2}}^{n+1, m} *\left(h_{N}^{n+1, m+1}-h_{b o t}\right)\right] \\
=\Delta t *\left[\frac{K_{N-\frac{1}{2}}^{n+1, m}-\left(K_{N+\frac{1}{2}}^{n+1, m} / 2\right)}{\Delta z}\right]-\left(\theta_{N}^{n+1, m}-\theta_{N}^{n}\right)+\Delta t * S_{i}^{n+1} \\
C_{N}^{n+1, m} * h_{N}^{n+1, m+1}-\frac{\Delta t}{(\Delta z)^{2}} * K_{N-\frac{1}{2}}^{n+1, m} * h_{N-1}^{n+1, m+1}+\frac{\Delta t}{(\Delta z)^{2}} *\left(K_{N-\frac{1}{2}}^{n+1, m}+K_{N+\frac{1}{2}}^{n+1, m}\right) * h_{N}^{n+1, m+1} \\
=C_{N}^{n+1, m} * h_{N}^{n+1, m}+\frac{\Delta t}{(\Delta z)^{2}} * K_{N+\frac{1}{2}}^{n+1, m} * h_{b o t}+\Delta t *\left[\frac{K_{N-\frac{1}{2}}^{n+1, m}-\left(K_{N+\frac{1}{2}}^{n+1, m} / 2\right)}{\Delta z}\right]-\left(\theta_{N}^{n+1, m}-\theta_{N}^{n}\right)+\Delta t * S_{i}^{n+1}
\end{gathered}
$$

Thus,

$$
\begin{aligned}
& \alpha_{N}=-\frac{\Delta t}{(\Delta z)^{2}} * K_{N-\frac{1}{2}}^{n+1, m} \\
& \beta_{N}=C_{N}^{n+1, m}+\frac{\Delta t}{(\Delta z)^{2}} *\left(K_{N-\frac{1}{2}}^{n+1, m}+K_{N+\frac{1}{2}}^{n+1, m}\right) \\
& \gamma_{N}=0 \\
& f_{N}=C_{N}^{n+1, m} * h_{N}^{n+1, m}+\frac{\Delta t}{(\Delta z)^{2}} * K_{N+\frac{1}{2}}^{n+1, m} * h_{b o t}+\Delta t *\left[\frac{K_{N-\frac{1}{2}}^{n+1, m}-\left(K_{N+\frac{1}{2}}^{n+1, m} / 2\right)}{\Delta z}\right]-\left(\theta_{N}^{n+1, m}-\theta_{N}^{n}\right)+\Delta t * S_{i}^{n+1}
\end{aligned}
$$


Bottom boundary condition $(\mathrm{i}=\mathrm{N})$ : free drainage $\left(h_{N}^{n+1, m+1}=h_{N+1}^{n+1, m+1}\right)$

From Eq. B.2, we have:

$$
\begin{gathered}
\left.C_{N}^{n+1, m} *\left(h_{N}^{n+1, m+1}-h_{N}^{n+1, m}\right)-\frac{\Delta t}{(\Delta z)^{2}} *\left[K_{N-\frac{1}{2}}^{n+1, m} *\left(h_{N-1}^{n+1, m+1}-h_{N}^{n+1, m+1}\right)-K_{N+\frac{1}{2}}^{n+1, m} * 0\right)\right] \\
=\Delta t *\left[\frac{K_{N-\frac{1}{2}}^{n+1, m}-K_{N+\frac{1}{2}}^{n+1, m}}{\Delta z}\right]-\left(\theta_{N}^{n+1, m}-\theta_{N}^{n}\right)+\Delta t * S_{i}^{n+1} \\
C_{N}^{n+1, m} * h_{N}^{n+1, m+1}-\frac{\Delta t}{(\Delta z)^{2}} * K_{N-\frac{1}{2}}^{n+1, m} * h_{N-1}^{n+1, m+1}+\frac{\Delta t}{(\Delta z)^{2}} *\left(K_{N-\frac{1}{2}}^{n+1, m}\right) * h_{N}^{n+1, m+1} \\
=C_{N}^{n+1, m} * h_{N}^{n+1, m}+\Delta t *\left[\frac{K_{N-\frac{1}{2}}^{n+1, m}-K_{N+\frac{1}{2}}^{n+1, m}}{\Delta z}\right]-\left(\theta_{N}^{n+1, m}-\theta_{N}^{n}\right)+\Delta t * S_{i}^{n+1}
\end{gathered}
$$

Thus,

$$
\begin{aligned}
& \alpha_{N}=-\frac{\Delta t}{(\Delta z)^{2}} * K_{N-\frac{1}{2}}^{n+1, m} \\
& \beta_{N}=C_{N}^{n+1, m}+\frac{\Delta t}{(\Delta z)^{2}} *\left(K_{N-\frac{1}{2}}^{n+1, m}\right)
\end{aligned}
$$




$$
\begin{aligned}
& \gamma_{N}=0 \\
& f_{N}=C_{N}^{n+1, m} * h_{N}^{n+1, m}+\Delta t *\left[\frac{K_{N-\frac{1}{2}}^{n+1, m}-K_{N+\frac{1}{2}}^{n+1, m}}{\Delta z}\right]-\left(\theta_{N}^{n+1, m}-\theta_{N}^{n}\right)+\Delta t * \mathrm{~S}_{i}^{\mathrm{n}+1}
\end{aligned}
$$

\author{
Soil thermal transport \\ $n$ : Time \\ $m$ : Iteration level \\ $i$ : Location \\ $N$ : Number of nodes \\ $\mathrm{T}$ : Temperature \\ $\Delta t$ : Time step \\ $\Delta z:$ The size of node \\ $C^{\prime}$ : Volumetric heat capacity of the soil \\ $L_{f}$ : Volumetric latent heat of freezing \\ $\theta_{I}$ : Volume ice content \\ $\rho_{I}$ : Ice density \\ $\lambda$ : Soil thermal conductivity
}




$$
\begin{gathered}
C_{i}^{\prime n+1, m} * \frac{T_{i}^{n+1, m+1}-T_{i}^{n}}{\Delta t}-\left[L_{f} * \rho_{I} *\left(\frac{d \theta_{I}}{d T}\right)_{i}^{n+1, m} * \frac{T_{i}^{n+1, m+1}-T_{i}^{n+1, m}}{\Delta t}\right]-L_{f} * \rho_{I} \frac{\left(\theta_{I}\right)_{i}^{n+1, m}-\left(\theta_{I}\right)_{i}^{n}}{\Delta t} \\
=\frac{\lambda_{i+1 / 2}^{n+1, m} *\left(\frac{T_{i+1}^{n+1, m+1}-T_{i}^{n+1, m+1}}{\Delta z}\right)-\lambda_{i-1 / 2}^{n+1, m} *\left(\frac{T_{i}^{n+1, m+1}-T_{i-1}^{n+1, m+1}}{\Delta z}\right)}{\Delta z} \text { (B. 4) }
\end{gathered}
$$

From Eq. B.4, we have:

$$
\alpha_{i} * T_{i-1}^{n+1, m+1}+\beta_{i} * T_{i}^{n+1, m+1}+\gamma_{i} * T_{i+1}^{n+1, m+1}=f_{i}
$$

Here,

$$
\begin{gathered}
\alpha_{i}=\frac{-\lambda_{i-1 / 2}^{n+1, m}}{(\Delta z)^{2}} \\
\beta_{i}=\left(\frac{C_{i}^{n+1, m}}{\Delta t}-\frac{L_{f} * \rho_{I} *\left(\frac{d \theta_{I}}{d T}\right)_{i}^{n+1, m}}{\Delta t}+\frac{\lambda_{i+1 / 2}^{n+1, m}}{(\Delta z)^{2}}-\frac{-\lambda_{i-1 / 2}^{n+1, m}}{(\Delta z)^{2}}\right) \\
\gamma_{i}=-\frac{\lambda_{i+1 / 2}^{n+1, m}}{(\Delta z)^{2}} \\
f_{i}=\frac{C_{i}^{\prime n+1, m}}{\Delta t} * T_{i}^{n}-\frac{L_{f} * \rho_{I} *\left(\frac{d \theta_{I}}{d T}\right)_{i}^{n+1, m}}{\Delta t} * T_{i}^{n+1 . m}+L_{f} * \rho_{I} \frac{\left(\theta_{I}\right)_{i}^{n+1, m}-\left(\theta_{I}\right)_{i}^{n}}{\Delta t}
\end{gathered}
$$


It can be expressed with a matrix form:

$\left(\begin{array}{ccccc}\beta_{1} & \gamma_{1} & 0 & 0 & 0 \\ \alpha_{2} & \beta_{2} & \gamma_{2} & 0 & 0 \\ \cdots & \cdots & \cdots & \cdots & \cdots \\ 0 & 0 & 0 & \alpha_{N} & \beta_{N}\end{array}\right) *\left[\begin{array}{c}T_{1}^{n+1, m+1} \\ T_{2}^{n+1, m+1} \\ \vdots \\ T_{N}^{n+1, m+1}\end{array}\right]=\left[\begin{array}{c}f_{1} \\ f_{2} \\ \vdots \\ f_{N}\end{array}\right]$

Top boundary condition (i=1): specified temperature $\boldsymbol{T}_{\text {surf }}$

From Eq. B.4 and by assuming $T_{i-1}^{n+1, m+1}=T_{\text {surf }}$, we still have:

$$
\alpha_{i} * T_{i-1}^{n+1, m+1}+\beta_{i} * T_{i}^{n+1, m+1}+\gamma_{i} * T_{i+1}^{n+1, m+1}=f_{i}
$$

Here,

$$
\begin{gathered}
\alpha_{i}=0 \\
\beta_{i}=\left(\frac{C_{i}^{n+1, m}}{\Delta t}-\frac{L_{f} * \rho_{I} *\left(\frac{d \theta_{I}}{d T}\right)_{i}^{n+1, m}}{\Delta t}+\frac{\lambda_{i+1 / 2}^{n+1, m}}{(\Delta z)^{2}}-\frac{-\lambda_{i-1 / 2}^{n+1, m}}{(\Delta z)^{2}}\right) \\
\gamma_{i}=-\frac{\lambda_{i+1 / 2}^{n+1, m}}{(\Delta z)^{2}}
\end{gathered}
$$




$$
f_{i}=\frac{C_{i}^{\prime n+1, m}}{\Delta t} * T_{i}^{n}-\frac{L_{f} * \rho_{I} *\left(\frac{d \theta_{I}}{d T}\right)_{i}^{n+1, m}}{\Delta t} * T_{i}^{n+1 . m}+L_{f} * \rho_{I} \frac{\left(\theta_{I}\right)_{i}^{n+1, m}-\left(\theta_{I}\right)_{i}^{n}}{\Delta t}+\frac{\lambda_{i-1 / 2}^{n+1, m}}{(\Delta z)^{2}} * T_{\text {surf }}
$$

\section{Bottom boundary condition $(\mathrm{i}=\mathrm{N})$ : specified temperature $\boldsymbol{T}_{\text {bot }}$}

From Eq. B.4, and by assuming $T_{i+1}^{n+1, m+1}=T_{b o t}$, we still have:

$$
\alpha_{i} * T_{i-1}^{n+1, m+1}+\beta_{i} * T_{i}^{n+1, m+1}+\gamma_{i} * T_{i+1}^{n+1, m+1}=f_{i}
$$

Here,

$$
\begin{gathered}
\alpha_{i}=\frac{-\lambda_{i-1 / 2}^{n+1, m}}{(\Delta z)^{2}} \\
\beta_{i}=\left(\frac{C_{i}^{\prime n+1, m}}{\Delta t}-\frac{L_{f} * \rho_{I} *\left(\frac{d \theta_{I}}{d T}\right)_{i}^{n+1, m}}{\Delta t}+\frac{\lambda_{i+1 / 2}^{n+1, m}}{(\Delta z)^{2}}-\frac{-\lambda_{i-1 / 2}^{n+1, m}}{(\Delta z)^{2}}\right) \\
\gamma_{i}=0 \\
\frac{C_{i}^{\prime n+1, m}}{\Delta t} * T_{i}^{n}-\frac{L_{f} * \rho_{I} *\left(\frac{d \theta_{I}}{d T}\right)_{i}^{n+1, m}}{\Delta t} * T_{i}^{n+1 . m}+L_{f} * \rho_{I} \frac{\left(\theta_{I}\right)_{i}^{n+1, m}-\left(\theta_{I}\right)_{i}^{n}}{\Delta t}-\frac{\lambda_{i+1 / 2}^{n+1, m}}{(\Delta z)^{2}} * T_{b o t}
\end{gathered}
$$


Bottom boundary condition $(\mathrm{i}=\mathrm{N})$ : specified heat flux $\boldsymbol{F}_{b o t}$, and it is usually zero.

From Eq. B.4, and by assuming $\lambda_{i+1 / 2}^{n+1, m} *\left(\frac{T_{i+1}^{n+1, m+1}-T_{i}^{n+1, m+1}}{\Delta z}\right)=0$, we still have:

$$
\alpha_{i} * T_{i-1}^{n+1, m+1}+\beta_{i} * T_{i}^{n+1, m+1}+\gamma_{i} * T_{i+1}^{n+1, m+1}=f_{i}
$$

Here,

$$
\begin{gathered}
\alpha_{i}=\frac{-\lambda_{i-1 / 2}^{n+1, m}}{(\Delta z)^{2}} \\
\beta_{i}=\left(\frac{C_{i}^{\prime n+1, m}}{\Delta t}-\frac{L_{f} * \rho_{I} *\left(\frac{d \theta_{I}}{d T}\right)_{i}^{n+1, m}}{\Delta t}-\frac{-\lambda_{i-1 / 2}^{n+1, m}}{(\Delta z)^{2}}\right) \\
f_{i}=\frac{C_{i}^{\prime n+1, m}}{\Delta t} * 0 \\
\gamma_{i}^{n}-\frac{L_{f} * \rho_{I} *\left(\frac{d \theta_{I}}{d T}\right)_{i}^{n+1, m}}{\Delta t} * T_{i}^{n+1 . m}+L_{f} * \rho_{I} \frac{\left(\theta_{I}\right)_{i}^{n+1, m}-\left(\theta_{I}\right)_{i}^{n}}{\Delta t}
\end{gathered}
$$

Celia, M. A., Bouloutas, E. T., and Zarba, R. L.: A general mass-conservative numerical solution for the unsaturated flow equation, Water.

Resour. Res., 26, 1483-1496, 
van Dam, J. C., Feddes, R. A.: Numerical simulation of infiltration, evaporation, and shallow groundwater levels with the Richards equation. J.

Hydro., 233, 72-85, 2000. 


\section{Supplement C}

\section{Estimation of DOC production rate}

According to Yurova et al (2008), the DOC production rate in pealands was provided at a value of $1.4^{*} 10^{-3} \mathrm{mg} \mathrm{g}^{-1} \mathrm{~h}^{-1}$. Due to the lack of DOC production rates for different land cover types and root fraction, we use the size of soil carbon pools which are highly linked to vegetation types to estimate the production rates for different types. The soil carbon pools on vegetation types are simulated by the Terrestrial Ecosystem Model (TEM, Zhuang et al. 2004) and the average values from 1980 to 2005 are listed in the Table C.1. Since TEM only simulated the region north of $55 \mathrm{~N}$ in this study, some vegetation types (such as tropical forests) are not listed in the Table C.1. The production rate $\left(1.4^{*} 10^{-3} \mathrm{mg} \mathrm{g}^{-1} \mathrm{~h}^{-1}\right)$ is assumed happened in a depth of wetland soil where the highest root fraction distributes. The DOC production rates for other vegetation types are assigned according to the ratio of their soil organic carbon pools to that of wetland. For example, boreal needleleaf evergreen forest has 10291.6 $\left(\mathrm{g} \mathrm{C}^{2} \mathrm{~m}^{2}\right)$ in its soil organic carbon pool. Thus, its DOC production rates (at depth which has the largest root fraction) is $1.4^{*} 10^{-3} *(10291.6 / 21170.4)=6.8 * 10^{-4} \mathrm{mg} \mathrm{g}^{-1}$ $\mathrm{h}^{-1}$. The TEM does not support the simulation for the mixed forests, woodland and wooded grassland. We assumed that mixed forests and woodland have the same DOC production rates to the average DOC production rate of evergreen needleleaf and deciduous needleleaf. Wooded grassland has the same rate as that of open shrublands. It is important to note that the peat density in Yurova et al., 2008 is usually between 0.02 and $0.06 \mathrm{~g} \mathrm{~cm}^{-3}$. Therefore, the final production rates should be reduced according to the ratio between the above peat density and the soil density in the model.

The soil organic carbon normally does not evenly distribute in soil profile and the root fraction also affects DOC production rates. Root fraction (R_f) of different vegetation types are estimated by (Zeng 2001):

$$
\text { R_f }=1-0.5 *(\exp (-1.0 * A * D)+\exp (-1.0 * B * D)) C .1
$$

Where D is the soil depth (meter, from the surface) and the values for A and B are listed in Table C.2. The DOC production rates at different soil depths should be adjusted according to their root fraction. For instance, evergreen needleleaf has the highest root faction at the top $1 \mathrm{~cm}$ soil layer $(0.0431)$ and the root fraction at a depth of $2 \mathrm{~cm}$ is 0.0408 , therefore the DOC production rate at $2 \mathrm{~cm}$ is only $94.6 \%$ $(0.0408 / 0.0431)$ of the surface layer. 
Table C.1 The Soil organic carbon and DOC production rates for different vegetation types.

\begin{tabular}{|c|c|c|c|c|c|}
\hline Vegetation types in the TEM simulation & $\begin{array}{c}\text { Soil Organic Carbon } \\
\left(\mathrm{g} \mathrm{C} / \mathrm{m}^{2}\right)\end{array}$ & $\begin{array}{c}\text { Vegetation types } \\
\text { in the DOC modeling }\end{array}$ & $\begin{array}{c}\text { DOC production rates } \\
\left(10^{-3} \mathrm{mg} \mathrm{g}^{-1} \mathrm{~h}^{-1}\right)\end{array}$ & \multicolumn{2}{|c|}{$\begin{array}{c}\text { A and B values in } \\
\text { Equation C.1 }\end{array}$} \\
\hline Bare Ground & 729.9 & Bare & 0.048268 & $10.74^{*}$ & $2.608^{*}$ \\
\hline Temperate Needleleaf Evergreen Forest & 6159.3 & $\begin{array}{c}\text { Evergreen Needleleaf } \\
\text { Forest }\end{array}$ & 0.407315 & 6.706 & 2.175 \\
\hline Boreal Needleleaf Evergreen Forest & 10291.6 & $\begin{array}{c}\text { Evergreen Needleleaf } \\
\text { Forest } \\
\text { (Used in this study) }\end{array}$ & 0.680584 & 6.706 & 2.175 \\
\hline Boreal Needleleaf Deciduous Forest & 8618.6 & $\begin{array}{c}\text { Deciduous Needleleaf } \\
\text { Forest }\end{array}$ & 0.569949 & 7.066 & 1.953 \\
\hline Temperate Broadleaved Deciduous Forest & 6801.8 & $\begin{array}{c}\text { Deciduous Broadleaf } \\
\text { Forest }\end{array}$ & 0.449803 & 5.990 & 1.955 \\
\hline Boreal Broadleaved Deciduous Forest & 8144.3 & $\begin{array}{c}\text { Feciduous Broadleaf } \\
\text { Forest }\end{array}$ & 0.538583 & 5.990 & 1.955 \\
\hline Broadleaved Evergreen Shrub & 3766.3 & Closed Shrublands & 0.249066 & 6.326 & 1.567 \\
\hline Broadleaved Deciduous Shrub & 3166.3 & Open Shrublands & 0.209388 & 7.718 & 1.262 \\
\hline C3 Grass & 3694.3 & Grasslands & 0.244304 & 10.74 & 2.608 \\
\hline C4 Grass & 3493.5 & $\begin{array}{c}\text { Grasslands } \\
\text { (Used in this study) }\end{array}$ & 0.231025 & 10.74 & 2.608 \\
\hline Boreal Herbaceous Wetland & 21170.4 & Wetland & 1.4 & $6.706^{*}$ & $2.175^{*}$ \\
\hline
\end{tabular}

*: there is no root distribution information for bare Ground and boreal Herbaceous Wetland. We used the values for grass and temperate needleleaf evergreen forest instead. 
Table C.2 Parameters used for estimating the root fraction in Equation C.1

\begin{tabular}{|c|c|c|}
\hline \multirow{2}{*}{ Vegetation Types } & \multicolumn{2}{|c|}{$\begin{array}{c}\text { A and B values in } \\
\text { Equation C.1 }\end{array}$} \\
\hline Evergreen Needleleaf & 6.706 & 2.175 \\
\hline Evergreen Broadleaf & 7.344 & 1.303 \\
\hline Deciduous Needleleaf & 7.066 & 1.953 \\
\hline Deciduous Broadleaf & 5.990 & 1.955 \\
\hline Mixed forest & 4.453 & 1.631 \\
\hline Woodland & 8.235 & 1.627 \\
\hline Wooded Grasslands & 7.604 & 2.300 \\
\hline Closed Shrublands & 6.326 & 1.567 \\
\hline Open Shrublands & 7.718 & 1.262 \\
\hline Grasslands & 10.74 & 2.608 \\
\hline Crop land (corn) & 5.558 & 2.614 \\
\hline
\end{tabular}

Yurova, A., Sirin, A., Buffam, I., Bishop, K., Laudon, H., 2008, Modeling the dissolved organic carbon output from a boreal mire using the convection-dispersion equation: Importance of representing sorption. Water Resources Research 44, W07411, doi:10.1029/2007WR006523.

Zeng, X. B., 2001: Global vegetation root distribution for land modeling. Journal of Hydrometeorology 2,525-530.

Zhuang, Q., J. M. Melillo, D. W. Kicklighter, R. G. Prinn, D. A. McGuire, P. A. Steudler, B. S. Felzer, and S. Hu. 2004. Methane fluxes between terrestrial ecosystems and the atmosphere at northern high latitudes during the past century: a retrospective analysis with a process-based biogeochemistry model. Global Biogeochemical Cycles 18: GB3010. 


\section{Supplement D}

$$
\begin{aligned}
& \frac{\partial\left(\theta_{I} C_{I}^{\prime \prime \prime}+\theta_{L} C^{\prime \prime \prime}\right)}{\partial \mathrm{t}}=\frac{\partial\left(\mathrm{q} C^{\prime \prime \prime}\right)}{\partial \mathrm{z}}+\frac{\partial\left(\theta_{L} D \frac{\partial C^{\prime \prime \prime}}{\partial z}\right)}{\partial \mathrm{z}}+\left(P^{\prime}\right)_{i}^{j+1}-\left(M^{\prime}\right)_{i}^{j+1}+\left(S^{\prime}\right)_{i}^{j+1}-\left(R^{\prime}\right)_{i}^{j+1} \\
& \frac{\left(\theta_{I}\right)_{i}^{j+1} C_{I}^{\prime \prime \prime}{ }_{i}^{j+1}-\left(\theta_{I}\right)_{i}^{j} C_{I}^{\prime \prime \prime}{ }_{i}^{j}+\left(\theta_{L}\right)_{i}^{j+1} C^{\prime \prime \prime}{ }_{i}^{j+1}-\left(\theta_{L}\right)_{i}^{j} C^{\prime \prime \prime}{ }_{i}^{j}}{\Delta \mathrm{t}}=\frac{\mathrm{q}_{\mathrm{i}-\frac{1}{2}}^{\mathrm{j}+1} C_{\mathrm{i}-\frac{1}{2}}^{\prime \prime \prime} \mathrm{q}_{\mathrm{i}+\frac{1}{2}}^{j+1} C_{i+\frac{1}{2}}^{\prime \prime \prime}}{\Delta \mathrm{z}}+\frac{1}{\Delta \mathrm{z}} *\left[\frac{\left(\theta_{L}\right)_{i-\frac{1}{2}}^{j+1} D_{i-\frac{1}{2}}^{j+1} *\left(C^{\prime \prime \prime}{ }_{i-1}^{j+1}-C^{\prime \prime \prime}{ }_{i}^{j+1}\right)}{\Delta z}-\frac{\left(\theta_{L}\right)_{i+\frac{1}{2}}^{j+1} D_{i+\frac{1}{2}}^{j+1} *\left(C^{\prime \prime \prime}{ }_{i}^{j+1}-C^{\prime \prime \prime}{ }_{i+1}^{j+1}\right)}{\Delta z}\right]+ \\
& \left(P^{\prime}\right)_{i}^{j+1}-\left(M^{\prime}\right)_{i}^{j+1}-\left(S^{\prime}\right)_{i}^{j+1}-\left(R^{\prime}\right)_{i}^{j+1}(\mathrm{D} .1)
\end{aligned}
$$

Also note that the subscription $i \pm \frac{1}{2}$ means the value at upper $(-)$ and lower $(+)$ interface of two adjacent nodes

The Eq. D.1 can be changed into:

$$
\begin{aligned}
& -\left(\frac{\mathrm{q}_{\mathrm{i}-\frac{1}{2}}^{\mathrm{j}+1}}{2 * \Delta \mathrm{z}}+\frac{\left(\theta_{L}\right)_{i-\frac{1}{2}}^{j+1} D_{i-\frac{1}{2}}^{j+1}}{(\Delta \mathrm{z})^{2}}\right) * C^{\prime \prime \prime}{ }_{i-1}^{j+1}+\left[\frac{\left(\theta_{L}\right)_{i}^{j+1}}{\Delta \mathrm{t}}-\frac{\mathrm{q}_{\mathrm{i}-\frac{1}{2}}^{\mathrm{j}+1}}{2 * \Delta \mathrm{z}}+\frac{\mathrm{q}_{\mathrm{i}+\frac{1}{2}}^{\mathrm{j}+1}}{2 * \Delta \mathrm{z}}+\frac{\left(\theta_{L}\right)_{i-\frac{1}{2}}^{j+1} D_{i-\frac{1}{2}}^{j+1}+\left(\theta_{L}\right)_{i+\frac{1}{2}}^{j+1} D_{i+\frac{1}{2}}^{j+1}}{(\Delta \mathrm{z})^{2}}\right] * C^{\prime \prime \prime}{ }_{i}^{j+1}+ \\
& {\left[\frac{\mathrm{q}_{\mathrm{i}+\frac{1}{2}}^{\mathrm{j}+1}}{2 * \Delta \mathrm{z}}-\frac{\left(\theta_{L}\right)_{\mathrm{i}+\frac{1}{2}}^{\mathrm{j}+1} \mathrm{D}_{\mathrm{i}+\frac{1}{2}}^{\mathrm{j}+1}}{(\Delta \mathrm{z})^{2}}\right]^{*} C^{\prime \prime \prime}{ }_{i+1}^{j+1}=\frac{-1 *\left(\theta_{I}\right)_{i}^{j+1} C_{I}^{\prime \prime \prime}{ }_{i}^{j+1}+\left(\theta_{I}\right)_{i}^{j} C_{I}^{\prime \prime \prime}{ }_{i}^{j}+\left(\theta_{L}\right)_{i}^{j}{ }^{\prime \prime \prime \prime}{ }_{i}^{j}}{\Delta \mathrm{t}}+\left(P^{\prime}\right)_{i}^{j+1}-\left(M^{\prime}\right)_{i}^{j+1}-\left(S^{\prime}\right)_{i}^{j+1}-\left(R^{\prime}\right)_{i}^{j+1} \text { (D.2) }}
\end{aligned}
$$

By defining: 


$$
\begin{gathered}
A=-\left(\frac{\mathrm{q}_{\mathrm{i}-\frac{1}{2}}^{\mathrm{j}+1}}{2 * \Delta \mathrm{z}}+\frac{\left(\theta_{L}\right)_{i-\frac{1}{2}}^{j+1} D_{i-\frac{1}{2}}^{j+1}}{(\Delta z)^{2}}\right) \\
B=\left[\frac{\left(\theta_{L}\right)_{i}^{j+1}}{\Delta \mathrm{t}}-\frac{\mathrm{q}_{\mathrm{i}-\frac{1}{2}}^{\mathrm{j}+1}}{2 * \Delta \mathrm{z}}+\frac{\mathrm{q}_{\mathrm{i}+\frac{1}{2}}^{\mathrm{j}+1}}{2 * \Delta \mathrm{z}}+\frac{\left(\theta_{L}\right)_{i-\frac{1}{2}}^{j+1} D_{i-\frac{1}{2}}^{j+1}+\left(\theta_{L}\right)_{i+\frac{1}{2}}^{j+1} D_{i+\frac{1}{2}}^{j+1}}{(\Delta z)^{2}}\right] \\
E=\frac{\mathrm{q}_{\mathrm{i}+\frac{1}{2}}^{\mathrm{j}+1}}{2 * \Delta \mathrm{z}}-\frac{\left(\theta_{L}\right)_{i+\frac{1}{2}}^{j+1} D_{i+\frac{1}{2}}^{j+1}}{(\Delta z)^{2}}
\end{gathered}
$$

The equation D. 2 can be also reorganized as:

$$
A * C^{\prime \prime \prime}{ }_{i-1}^{j+1}+\mathrm{B} * C^{\prime \prime \prime}{ }_{i}^{j+1}+E * C^{\prime \prime \prime}{ }_{i+1}^{j+1}=\frac{-1 *\left(\theta_{I}\right)_{i}^{j+1} C_{I}^{\prime \prime \prime}{ }_{i}^{j+1}+\left(\theta_{I}\right)_{i}^{j} c_{I}^{\prime \prime \prime}{ }_{i}^{j}+\left(\theta_{L}\right)_{i}^{j} C^{\prime \prime \prime \prime}{ }_{i}^{j}}{\Delta \mathrm{t}}+\left(P^{\prime}\right)_{i}^{j+1}-\left(M^{\prime}\right)_{i}^{j+1}-\left(S^{\prime}\right)_{i}^{j+1}-\left(R^{\prime}\right)_{i}^{j+1} \text { (D.3) }
$$

The production item $\left(\left(P^{\prime}\right)_{i}^{j+1}\right): p_{i}^{j+1} * \rho_{b}$

The mineralization item $\left(\left(M^{\prime}\right)_{i}^{j+1}\right): M_{i}^{j+1} *\left(\theta_{L}\right)_{i}^{j+1} * C_{i}^{\prime \prime \prime}{ }_{i}^{j+1}$

Absorption[desorption] item $\left(\left(S^{\prime}\right)_{i}^{j+1}\right): \frac{\rho_{b^{*}}\left(S 1_{i}^{j+1}-S 1_{i}^{j}\right)+\rho_{b^{*}}\left(S 2_{i}^{j+1}-S 2_{i}^{j}\right)}{\Delta \mathrm{t}}$

Transfer from soil solution to overland flow item: $\left(R^{\prime}\right)_{i}^{j+1}$ : This item has a value large than zero only on the top node (i=1), all others are zero. All the five items have the unit in $\mathrm{g} / \mathrm{cm}^{3} \mathrm{~s}$

Where $S 1_{i}^{j+1}, S 2_{i}^{j+1}$ are type1 and type 2 PDCO at the current time step and they can be expressed as: 


$$
\begin{gathered}
S 1_{i}^{j+1}=f * K d * C^{\prime \prime \prime}{ }_{i}^{j+1} \\
S 2_{i}^{j+1}=S 2_{i}^{j}+\Delta S 2_{i}^{j} \\
\Delta S 2_{i}^{j}=\left[a\left((1-f) * K_{d} * C^{\prime \prime \prime}{ }_{i}^{j+1}\right)-a * S 2_{i}^{j}\right] * \Delta t
\end{gathered}
$$

By incorporating production, mineralization and absorption/desorption items, Eq. D.3 can be also reorganized as:

$$
\begin{gathered}
A * C^{\prime \prime \prime}{ }_{i-1}^{j+1}+\left[B+\left(\frac{\rho_{b} * f * K d}{\Delta t}+\rho_{b} * a *(1-f) * K_{d}\right)+M_{i}^{j+1} *\left(\theta_{L}\right)_{i}^{j+1}\right] * C^{\prime \prime \prime}{ }_{i}^{j+1}+E * C^{\prime \prime \prime}{ }_{i+1}^{j+1}=\frac{\left.-1 *\left(\theta_{I}\right)_{i}^{j+1} C_{I}^{\prime \prime \prime}{ }_{i}^{j+1}+\left(\theta_{I}\right)_{i}^{j} C_{I}^{\prime \prime \prime}{ }_{i}^{j+\left(\theta_{L}\right.}\right)_{i}^{j} C^{\prime \prime \prime \prime}{ }_{i}^{j}}{\Delta \mathrm{t}}+p_{i}^{j+1} * \\
\rho_{b}+\frac{\rho_{b^{*}}\left(S 1_{i}^{j}\right)}{\Delta t}+S 2_{i}^{j} * a * \rho_{b}-\left(R^{\prime}\right)_{i}^{j+1} \text { (D.4) }
\end{gathered}
$$

\section{Boundary conditions}

\section{Top boundary}

The specified flux is used as the top boundary and we considered three situations on the top boundary:

1. No ponding water and rainfall $>0$

$$
\begin{gathered}
C_{\text {top }}^{\prime \prime \prime}=0 \\
\mathrm{q}_{\text {top }}^{\mathrm{j}+1}=\text { rainfall }
\end{gathered}
$$


2. No ponding water and rainfall $=0$

$$
\begin{aligned}
C_{\text {top }}^{\prime \prime \prime} & =0 \\
\mathrm{q}_{\text {top }}^{j+1} & =0
\end{aligned}
$$

3. Ponding water

$$
\begin{gathered}
C_{\text {top }}^{\prime \prime \prime}=C_{\text {overland }}^{\prime \prime \prime} \\
\mathrm{q}_{\text {top }}^{\mathrm{j}+1}=\mathrm{Q}_{\mathrm{in}}
\end{gathered}
$$

$C_{\text {overland }}$ and $\mathrm{Q}_{\text {in }}$ are the overland DOC concentration and infiltration flow rate at the top, respectively.

On the top (i=1), we have:

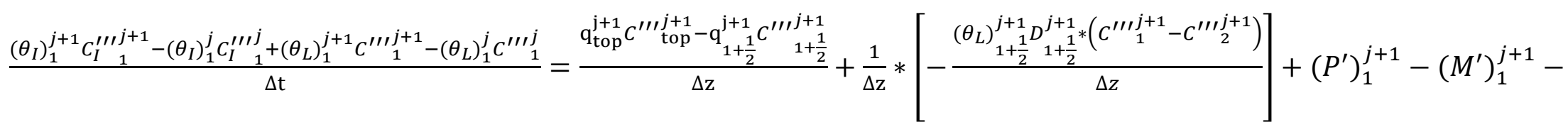

$$
\begin{aligned}
& \left(S^{\prime}\right)_{1}^{j+1}-\left(R^{\prime}\right)_{1}^{j+1}(\mathrm{D} .5)
\end{aligned}
$$

Note that $C_{1+\frac{1}{2}}^{\prime \prime \prime}=\frac{C^{\prime \prime \prime}{ }_{1}^{j+1}+C^{\prime \prime \prime}{ }_{2}^{j+1}}{2}$ and by incorporating production, mineralization and absorption/desorption items, Eq. D.5 can be reorganized as: 


$$
\begin{gathered}
{\left[\left[\frac{\left(\theta_{L}\right)_{1}^{j+1}}{\Delta \mathrm{t}}+\frac{\mathrm{q}_{1+\frac{1}{2}}^{\mathrm{j}+1}}{2 * \Delta \mathrm{z}}+\frac{\theta_{1+\frac{1}{2}}^{j+1} D_{1+\frac{1}{2}}^{j+1}}{(\Delta z)^{2}}\right]+\left(\frac{\rho_{b} * f * K_{d}}{\Delta t}+\rho_{b} * a *(1-f) * K_{d}\right)+M_{1}^{j+1} *\left(\theta_{L}\right)_{1}^{j+1}\right] * C_{1}^{\prime \prime \prime}{ }_{1}^{j+1}+E * C_{2}^{\prime \prime \prime}{ }_{2}^{j+1}=} \\
\frac{-1 *\left(\theta_{I}\right)_{1}^{j+1} C_{I}^{\prime \prime \prime}{ }_{1}^{j+1}+\left(\theta_{I}\right)_{1}^{j} C_{I}^{\prime \prime \prime}{ }_{1}^{j}+\left(\theta_{L}\right)_{1}^{j} C^{\prime \prime \prime}{ }_{1}^{j}}{\Delta \mathrm{t}}+\frac{\left(\theta_{L}\right)_{1}^{j} C^{\prime \prime \prime}{ }_{1}^{j}}{\Delta \mathrm{t}}+\frac{\mathrm{q}_{\mathrm{top}}^{\mathrm{j}+1} C^{\prime \prime \prime \prime}{ }_{\mathrm{top}}^{j+1}}{\Delta \mathrm{t}}+p_{1}^{j+1} * \rho_{b}+\frac{\rho_{b} *\left(S 1_{1}^{j}\right)}{\Delta t}+S 2_{1}^{j} * a * \rho_{b}-\left(R^{\prime}\right)_{1}^{j+1} \text { (D.6) }
\end{gathered}
$$

\section{Bottom boundary}

The specified flux is again used as the bottom boundary:

$$
\begin{gathered}
C_{\text {bottom }}^{\prime \prime \prime}=C_{\text {bottom }}^{j+1} \\
\mathrm{q}_{\text {bottom }}^{\mathrm{j}+1}=\mathrm{q}_{\text {drainage }}^{\mathrm{j}+1}
\end{gathered}
$$

at the bottom $(\mathrm{i}=\mathrm{n})$, we have:

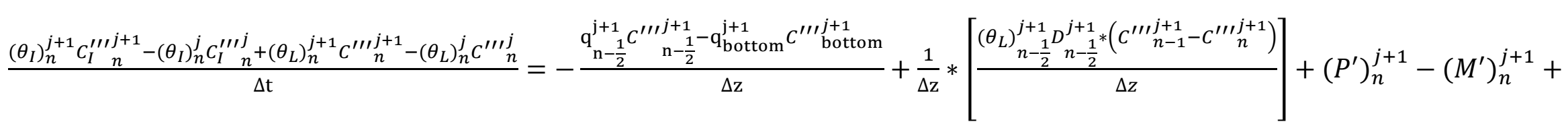

$$
\begin{aligned}
& \left(S^{\prime}\right)_{n}^{j+1}-\left(R^{\prime}\right)_{n}^{j+1}(\mathrm{D} .7)
\end{aligned}
$$

Note that $C_{\mathrm{n}+\frac{1}{2}}^{\prime \prime \prime}=\frac{C_{\mathrm{n}}^{\prime \prime \prime}{ }_{\mathrm{n}}^{j+1}+C^{\prime \prime \prime}{ }_{\mathrm{n}-1}^{j+1}}{2}$ and by incorporating production, mineralization and absorption/desorption items, the Eq. D.7 can be 
reorganized as:

$\left[-\frac{\mathrm{q}_{\mathrm{n}-\frac{1}{2}}^{\mathrm{j}+1}}{2 * \Delta \mathrm{z}}-\frac{\left(\theta_{L}\right)_{n-\frac{1}{2}}^{j+1} D^{j+1} \frac{1}{2}}{\Delta z^{2}}\right] * C_{n-1}^{\prime \prime \prime}+\left[\left[\frac{\left(\theta_{L}\right)_{n}^{j+1}}{\Delta \mathrm{t}}-\frac{\mathrm{q}_{\mathrm{n}+\frac{1}{2}}^{\mathrm{j}+1}}{2 * \Delta \mathrm{z}}+\frac{\left(\theta_{L}{ }_{n-\frac{1}{2}}^{j+1} D_{n-\frac{1}{2}}^{j+1}\right.}{(\Delta z)^{2}}\right]+\left(\frac{\rho_{b} * f * K_{d}}{\Delta t}+\rho_{b} * a *(1-f) * K_{d}\right)+M_{n}^{j+1} *\left(\theta_{L}\right)_{n}^{j+1}\right] * C_{n}^{\prime \prime \prime \prime}{ }_{n}^{j+1}=$

$\frac{-1 *\left(\theta_{I}\right)_{n}^{j+1} C_{I}^{\prime \prime \prime}{ }_{n}^{j+1}+\left(\theta_{I}\right)_{n}^{j} C_{I}^{\prime \prime \prime}{ }_{n}^{j}+\left(\theta_{L}\right)_{n}^{j} C^{\prime \prime \prime}{ }_{n}^{j}}{\Delta \mathrm{t}}-\frac{\mathrm{q}_{\text {bottom }}^{\mathrm{j}+1} C_{\text {bottom }}^{j+1}}{\Delta \mathrm{t}}+p_{n}^{j+1} * \rho_{b}+\frac{\rho_{b} *\left(S 1_{n}^{j}\right)}{\Delta t}+S 2_{n}^{j} * a * \rho_{b}-\left(R^{\prime}\right)_{n}^{j+1}$ (D.8) 


\section{Supplement E}

\section{Channel width and depth}

The stream network is determined based on DEM dataset. Since the spatial resolution in this study is large $(4 \mathrm{~km})$, one important issue in making channel network is zero slopes in channel grids which may result in no drain. We use the channel elevation checking and smoothing tool proposed by England et al (2007) in generating channel network.

Channel cross-section geometry is specified as rectangular throughout the stream network. The channel geometric characteristics (width, depth, cross-section area) are assigned to each channel segment based on the depth-discharge relationship. For the Yukon River Region, drainage area and bank full discharge is related to each other by (Emmett., 1972):

$$
Q_{B}=27.1 * D A^{0.79}(\mathrm{E} .1)
$$

Where $Q_{B}$ is discharge of stream at bank full stage $\left(\mathrm{L}^{3} / \mathrm{T}\right) ; D A$ is drainage area $\left(\mathrm{L}^{2}\right)$. Next, bank full width $W_{B}$, and depth $D_{B}$ are approximated by:

$$
\begin{gathered}
W_{B}=1.7 * Q_{B}{ }^{0.54}(\text { E. } 2) \\
D_{B}=0.53 * Q_{B}{ }^{0.3}(\text { E. } 3)
\end{gathered}
$$

Manning's friction coefficients are assigned to each channel grid based on published values by Bedient and Huber (1988).

\section{Soil bulk density of overland flow}

We use sediment concentration measurement at the USGS gauge station (USGS $15476000)$ to estimate the soil bulk density in overland flow. The station provides the sediment concentration data from 1953 to 1966 . The monthly average values at the gauge station are used. The unit reported at the gauge station is $\mathrm{mg} / \mathrm{L}$ and we converted into $\mathrm{g} / \mathrm{cm}^{3}$. The Figure E.1 shows its variations: 


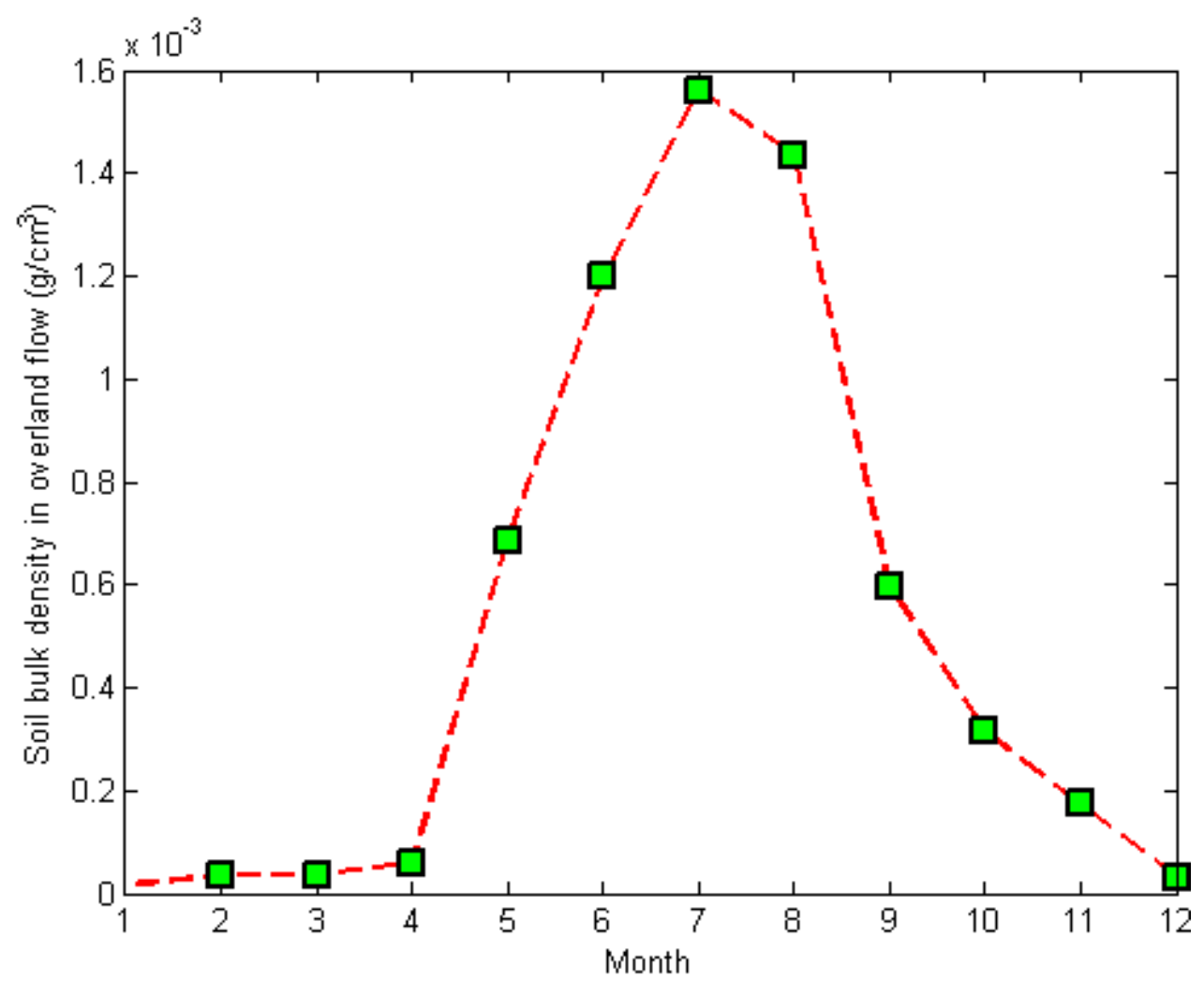

Fig. E.1 The month average soil bulk density in the overland flow used in this study.

Emmett, W. W.: The Hydraulic geometry of some Alaska streams south the Yukon River. Open-file Report, USGS, 1972.

England, J., Velleux, M., and Julien, P.: Two-dimensional simulations of extreme floods on a large watershed, J. Hydro., 347, 229-241, 2007.

Bedient, P.B., and Huber, W.C.: Floodplain hydraulics. Hydrology and Floodplain Analysis. Addison-Wesley, Reading, Massachusetts, 455-520, 1988. 


\section{Supplement F}

The streamflow data was required from the USGS gauge station 15484000. In the next two figures, we showed the streamflow comparison in 1976 and 2004, respectively:

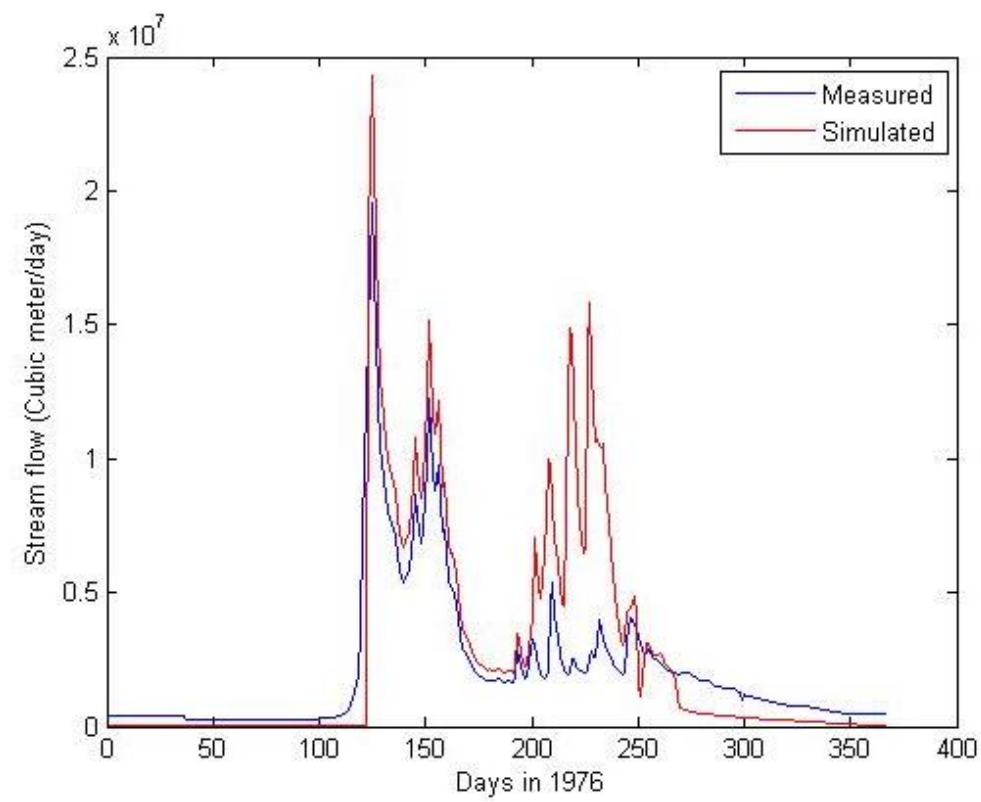

Fig. F.1 Comparison between measured and simulated streamflow in 1976.

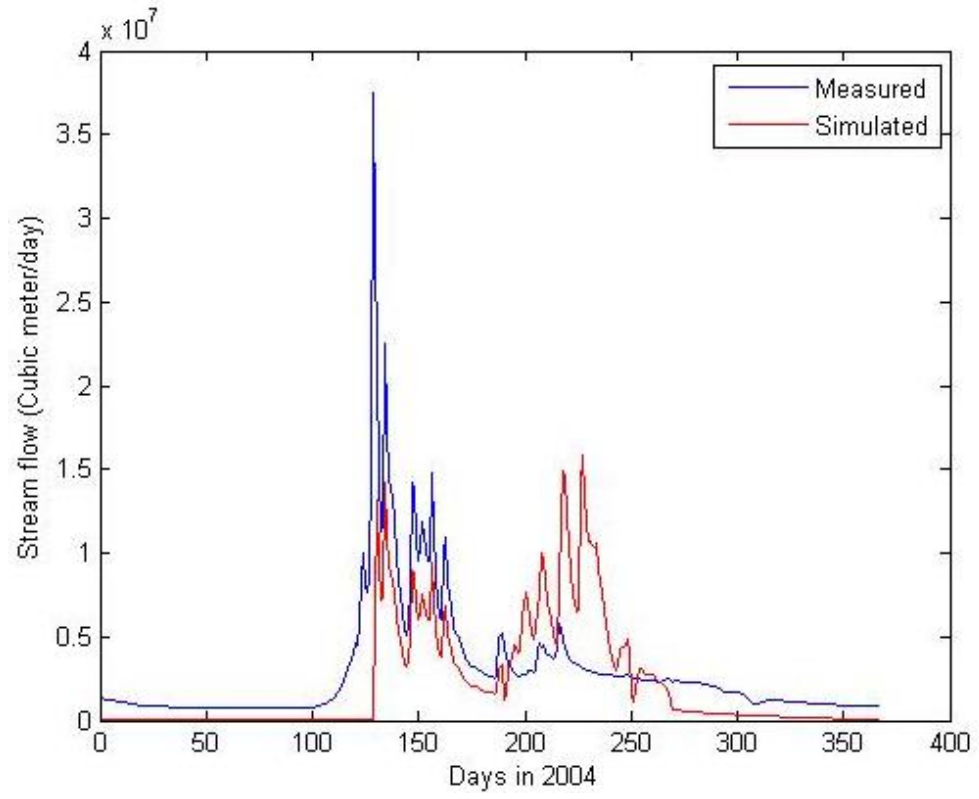

Fig. F.2 Comparison between measured and simulated streamflow in 2004.

From the above comparison, the spring flood mainly due to snow melting is well captured by the model. However, the model tended to overestimate in fall, suggesting that our model tends to yield high soil moisture.

The DOC comparison was shown in the following two figures. 


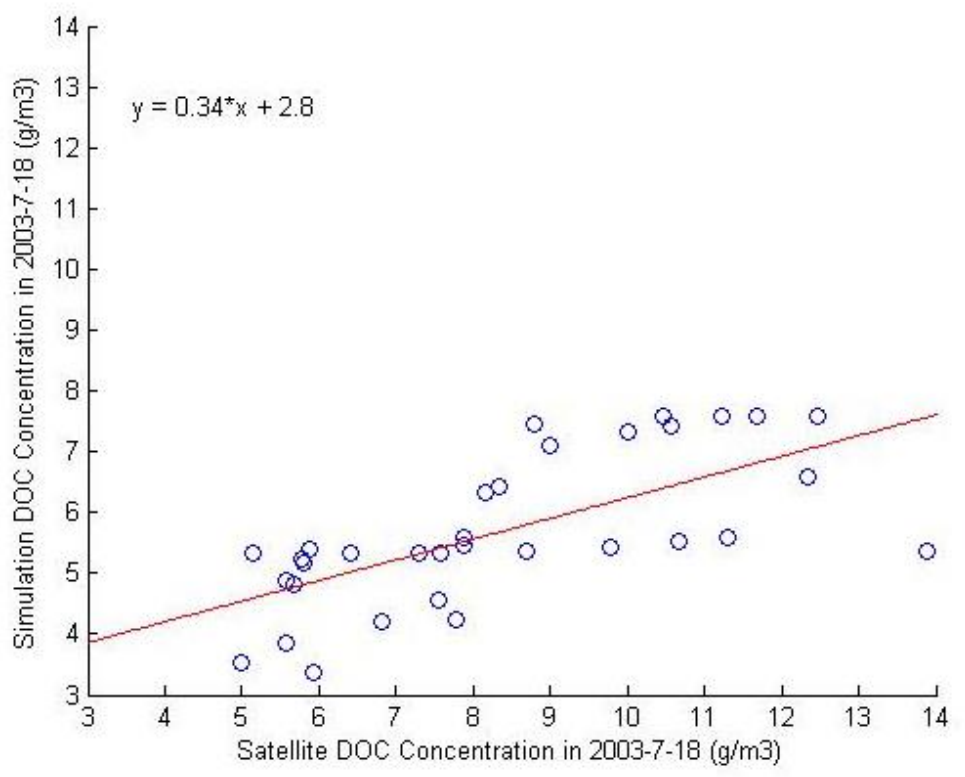

Fig. F.3 Comparison between remotely sensed and simulated DOC concentration on 2003-7-18.

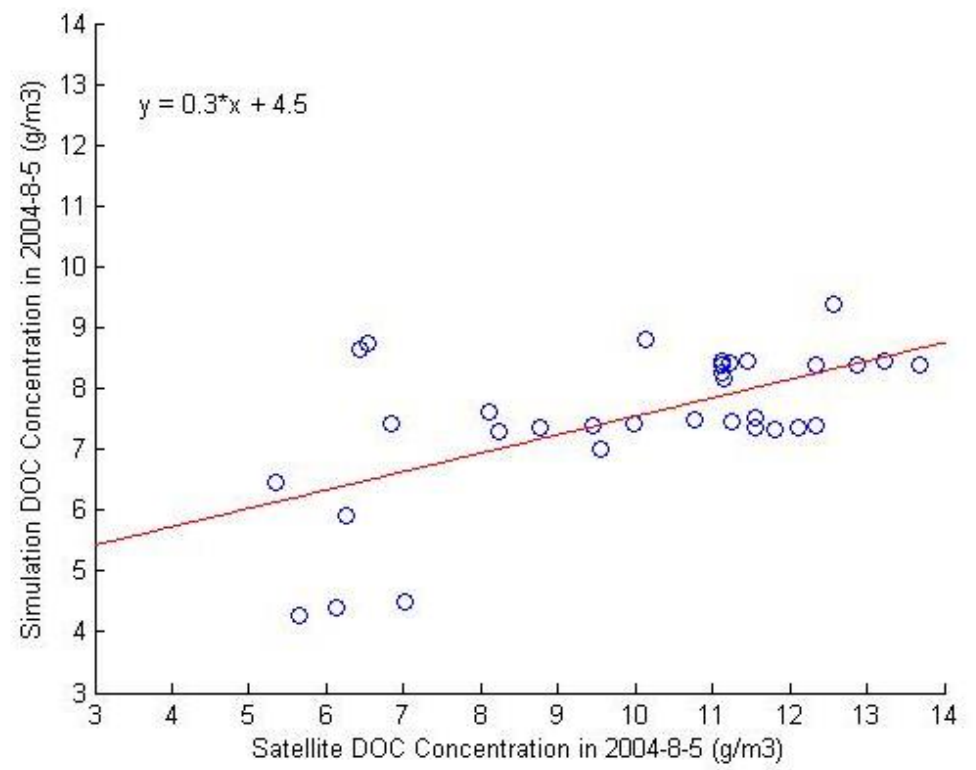

Fig. F.4 Comparison between remotely sensed and simulated DOC concentration on 2004-8-5.

Although the correlation is good for the data in the Figure F. $3(0.67)$ and $4(0.61)$, respectively, the model usually underestimates the DOC concentration. The modelled higher streamflow can partially explain the lower DOC concentrations. 